\title{
Finite-element simulation of sheet-forming processes with the help of contact elements on small-scale workstations
}

\author{
P.T. Vreede ${ }^{a, *}$, B.D. Carleer ${ }^{\mathrm{b}}$, M.F.M. Louwes ${ }^{\mathrm{b}}$, J. Huétink ${ }^{\mathrm{b}}$ \\ ${ }^{a}$ Hoogovens Groep Steelworks R\&D, P.O. Box 10000, 1970 CA IJmuiden, Netherlands

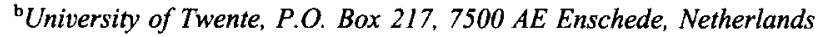

\section{Industrial Summary}

In this paper the development of an implicit code is illustrated. The sheet elements as well as the contact description need some special attention. Further, a connection with commercially available CAD packages is essential for a user-friendly range of application. Examples of a simulation of an automotive product and a multi-step deep-drawing process are given. To widen the scope, a start is made with explicit methods with emphasis on contact formulation.

\section{Sheet modelling}

If 3-dimensional theory is used to model thin sheets, the solution with solid 3-dimensional elements is plagued by numerical problems such as ill conditioning. In thin-sheet metal forming the deformation due to stretching dominates the deformation due to bending and shearing. With this assumption, membrane elements can be applied successfully. The simplest element amongst the membrane elements is the triangular constant-strain membrane element, which is used in the finite-element code.

The orientations of global and local coordinate systems are depicted in Fig. 1. The vector $e_{3}$ is located perpendicular to the element plane. The relationship between the local and global basis vectors and between the local and global displacements can be found from:

$$
e_{i}=R_{i \alpha} b_{\alpha} \quad u_{i}^{\text {loc }}=R_{i \alpha} u_{\alpha}^{\text {glob }}
$$

In this equation, $R_{i \alpha}$ are the components of an orthogonal matrix [R], whilst $\{\boldsymbol{u}\}_{\text {loc }}$ is defined as the column vector of all nodal displacement components related to the local (element) basis. The strain at every point within an element can be defined by the

\footnotetext{
* Corresponding author.
} 

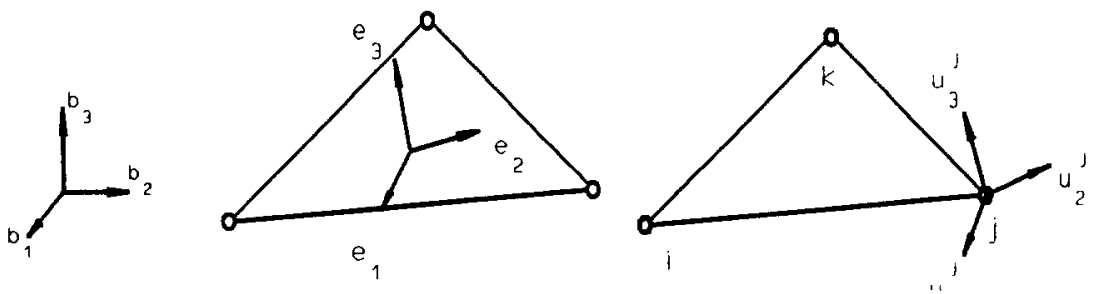

Fig. 1. Global and local coordinate system.

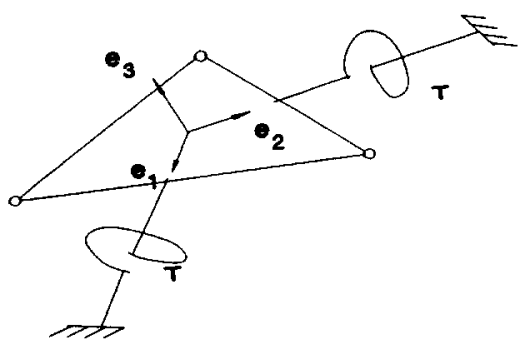

Fig. 2. Location of the torsion stiffness.

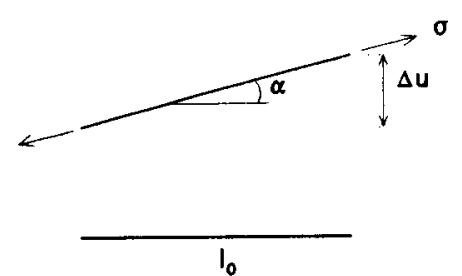

Fig. 3. A line element.

three strain components that contribute to internal work.

$$
\{\varepsilon\}=\left\{\begin{array}{lll}
\varepsilon_{x} & \varepsilon_{y} & \varepsilon_{x y}
\end{array}\right\}^{\mathrm{T}}=\sum_{m}^{N}\left[\boldsymbol{B}_{m}\right] \cdot\{\boldsymbol{u}\}_{\mathrm{loc}}^{m}=\sum_{m}^{N}\left[\boldsymbol{B}_{m}\right] \cdot[\boldsymbol{R}] \cdot\{\boldsymbol{u}\}_{\text {glob }}^{m},
$$

with a typical matrix $\left[\boldsymbol{B}_{m}\right]$ given by

$$
\left[\boldsymbol{B}_{m}\right]=\left[\begin{array}{cccc}
N_{. x} & 0 & 0 & \cdots \\
0 & N_{, y} & 0 & \cdots \\
N_{, y} & N_{, x} & 0 & \cdots
\end{array}\right]
$$

$N_{. x}$ is the shape function derived with respect to the local $x$-direction. It may be observed that the displacements perpendicular to the element do not contribute to internal work. Therefore a node of a single element will not have any stiffness in the normal direction. If a flat sheet is modelled by a collection of these elements, the stiffness matrix will be badly conditioned and possibly can not be inverted.

A satisfying solution is found by adding two torsion stiffnesses as depicted in Fig. 2. A small torsion stiffness stabilizes the calculation and has hardly any effect on the results. The application of the torsion springs can be regarded as a pre-stress condition in the membrane. This will be illustrated by a 2-dimensional example. Suppose a line element with length $l_{0}$ has a pre-stress $\sigma$, see Fig. 3. If the relative 
displacement is $u$, then angle $\alpha$ equals $u / l_{0}$. Due to the displacement $u$, an elongation $\varepsilon$ of the line element is

$$
\varepsilon=\frac{\left(l_{0} / \cos \alpha\right)-l_{0}}{l_{0}} \simeq \frac{l_{0}+(1 / 2) l_{0} \alpha^{2}-l_{0}}{l_{0}}=\frac{1}{2} \alpha^{2}=\frac{1}{2}\left[\frac{u}{l_{0}}\right]^{2} .
$$

The virtual power due to the displacement $u$ becomes

$$
\delta W=\int_{V} \sigma \frac{u}{l_{0}^{2}} \delta u \mathrm{~d} V
$$

In the present element a torsion spring is used. Suppose that the stiffness of the spring is $T$, then the moment $M$ is $\alpha T$. The virtual power becomes

$$
\delta W=\int_{V} M \delta \alpha \mathrm{d} V=\int_{V} \frac{u}{l_{0}} T \frac{\delta u}{l_{0}} \mathrm{~d} V .
$$

From the results of Eqs. (5) and (6) it can be concluded that the value of the torsion stiffness can be regarded as a pre-stress of value $T$.

Implementation can take place easily by introducing two fictive strain components $\varepsilon_{x z}^{*}$ and $\varepsilon_{y z}^{*}$. With the help of the $B$-matrix these strains are obtained from rotations of the element perpendicular to the element plane. By adding terms to the respective diagonal term in the constitutive tensor, the virtual power increases when the element rotates in plane, thus preventing the occurrence of zero energy modes.

\section{Material modelling}

Much research effort has been devoted to model the material behaviour during plastic deformation. Bulk material is usually regarded as behaving isotropically and the von Mises criterion gives an acceptable description of the elastic-plastic transition. For cold-rolled sheet metal this description no longer holds and a planar isotropic yield criterion is a substantial improvement compared to the von Mises yield criterion.

However, earing in a rotationally symmetric product indicates that the in-plane properties vary slightly as a function of the angle with respect to the rolling direction. When one chooses to resort to a fully anisotropic description, the original orthogonal axes may no longer remain orthogonal during deformation: a profound description has not yet been found. Linked up with this problem is the way hardening is modelled. The two extreme hardening models are isotropic hardening and kinematic hardening. It can be concluded from the Bauschinger effect that none of these models covers reality satisfactorily and it is likely that a mixture of these hardening models may give an improvement. A paper covering this topic related to experiments is in preparation by the present authors. In the constitutive model the yield condition is given by the inner yield surface, whereas the hardening rate is dependent on the distance between 


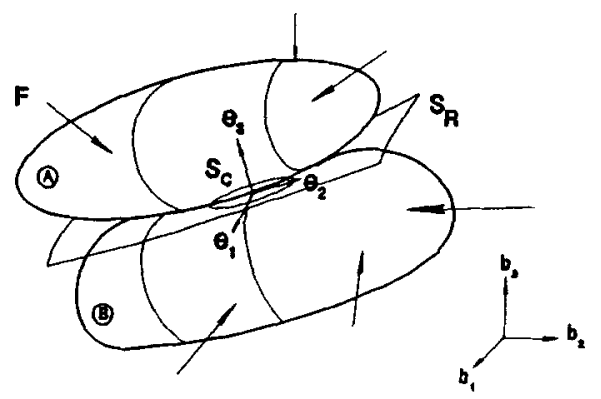

Fig. 4. Contacting bodies $A$ and $B$ with contact surface $S_{\mathrm{C}}$ and reference surface $S_{\mathrm{R}}$.

the inner yield surface and the outer (fictive) yield surface. The size of the outer yield surface (re)starts growing when the inner surface touches the outer surface with a rate according to the tensile test. A power law is used for the relationship between the hardening rate and the distance between both surfaces [1,2]. A similar theory has been proposed in [3-5]. Variations in the shape of the yield surface have been studied thoroughly by Vegter [6]. In the examples of this paper planar isotropic yielding and isotropic hardening is used.

\section{Modelling of contact}

Consider two bodies $A$ and $B$ and a contact surface $S_{\mathrm{C}}$ (Fig. 4) with the points $x^{A}$ and $x^{B}$ located on bodies $A$ and $B$ respectively.

Define a local orthonormal co-rotating coordinate system $\left(\boldsymbol{e}_{1}, \boldsymbol{e}_{2}, \boldsymbol{e}_{3}\right)$. The relative surface velocity, $d_{i}$, is defined as the difference between the velocities of the surfaces $S_{A}$ and $S_{B}$ with respect to the local coordinate system $e_{i}$

$$
d_{i}=\dot{x}_{i}^{A}-\dot{x}_{i}^{B}=\Delta \dot{x}_{i}
$$

Contact stresses are considered to be traction forces acting on the surface $S$. The normal stress component is $\tau_{3}$, whereas $\tau_{1}$ and $\tau_{2}$ are the shear stress components. Taking time derivatives of $\tau$ yields

$$
\dot{\tau}==\dot{\tau}_{i} \boldsymbol{e}_{i}+\tau_{i} \dot{\boldsymbol{e}}_{i} .
$$

It can be proven that the Jaumann stress tensor $\tau^{\nabla}$ can be expressed by the material rate of change of the stress components

$$
\tau^{\nabla}=\dot{\tau}_{i} \boldsymbol{e}_{\boldsymbol{i}}
$$

Friction is usually regarded to be an irreversible process only: however, it has a reversible part as well. The latter is caused by elastic deformations at the local contact spots (Fig. 5). For simulations of sheet-metal forming processes the reversible part not only has a physical meaning, it also stabilizes a calculation, especially during start-up. 


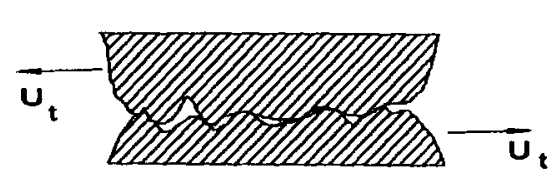

(a)

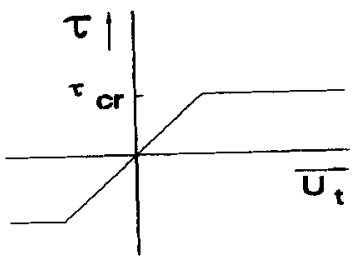

(b)

Fig. 5. Elastic response of the contact surface due to a tangential displacement.

A small tangential displacement $\left(U_{t}\right)$ will give a tangential stress, proportional to this displacement, see Fig. 5(b). This linear elastic contact behaviour results in

$$
\tau^{\nabla}=\underline{E}^{\mathrm{C}} \cdot \boldsymbol{d}^{\mathrm{rv}}
$$

where $d^{\text {rv }}$ is the reversible relative velocity. The stiffness tensor $\underline{E}^{\mathrm{C}}$ has a diagonal form. After a critical level of the tangential stress $\left(\tau_{\mathrm{cr}}\right)$ has been reached (Fig. $5(\mathrm{~b})$ ), the bodies slide relative to each other. In this case it is assumed that the relative velocity can be decomposed into a reversible part $d^{\text {rv }}$ and an irreversible part $d^{\text {ir }}$.

$$
d=d^{\mathrm{rv}}+d^{\mathrm{ir}}
$$

Substituting (11) into (10) results in

$$
\tau^{\nabla}=\underline{E}^{\mathrm{C}} \cdot\left(d-d^{\mathrm{ir}}\right)
$$

In the situation in which there is no contact, the stiffness between the bodies vanishes. In this case the stiffness tensor $\underline{E}^{\mathrm{C}}$ is set to zero.

The stick-slip constitutive behaviour is introduced in a way which is analogue to the description of elasto-plasticity with non-associated flow. In the case of contact with a possibility of frictional sliding, stick or slip will be governed by the function $\phi^{\mathrm{C}}(\tau)$

$$
\phi^{\mathrm{c}}=\tau_{1}^{2}+\tau_{2}^{2}-\mu^{2} \tau_{3}^{2},
$$

where $\mu$ is the coefficient of friction.

Similar to the way elasto-plastic constitutive relations are derived, the equations for contact behaviour can be found. A more detailed description can be found in [7]. In nearly all sheet-metal forming processes, lubricants are applied to prevent wear due to metal-to-metal contact and to reduce friction. In applications with elevated pressures, hydrostatic effects will develop and a description with a constant Coulomb friction is no longer satisfactory. The influence of viscosity, sliding velocity and pressure on the friction coefficient is often represented by a Stribeck curve. The effect of initial surface roughness on friction in conjunction with the Stribeck curve has been studied extensively by Emmens [8] and Emmens Montfort [9]. 


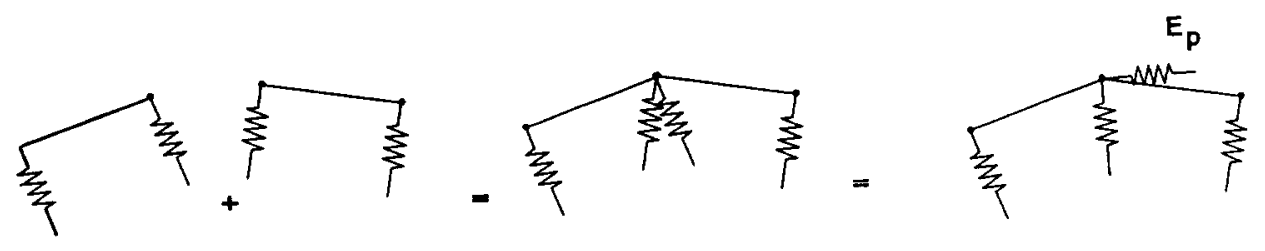

Fig. 6. Assembling of the contact elements.

During the forming stage the surface conditions may change dramatically, which in turn results in a different contact behaviour [10]: this effect is being studied currently by the tribology group at the University of Twente. When results become available, implementation in the contact elements will take place. The necessity to store historical information (elastic or plastic, amount of deformation, etc) gives rise to develop the special contact elements. Storage takes place similarly as in common finite elements.

\section{Pitfalls}

Suppose that a strongly curved zone in the contact surface is discretized with a relatively small number of contact elements. As a direct consequence, a relatively large angle between two adjacent elements is found.

Keeping in mind that a contact element is modelled by springs, some interference between the elements is found when a reference coordinate system at the element level is used. In two-dimensional space, this effect can be explained with the help of Fig. 6. Only springs in the normal direction are considered. After assembling the elements a parasitic stiffness arises, resulting in an extra resistance of the bulk or sheet material to motion around a curved zone.

This problem is solved by applying a local coordinate system at the nodal level instead of a coordinate system at element level. The nodal reference coordinate system can be calculated by interpolation of the element coordinate systems of neighbouring elements:

$$
\boldsymbol{e}_{i}^{\text {node }}=\frac{1}{N} \sum \boldsymbol{e}_{i}^{\text {elem } n} \quad n=1 \ldots N .
$$

The number of elements $N$ is connected to the relevant node.

\section{Numerical verification}

To validate the FE approximation, consider a strip of sheet metal clamped between the die and the blank-holder. When the strip is pulled out, a horizontal force $F_{\mathrm{H}}$ must be applied to move the strip. $F_{\mathrm{H}}=2 \mu F_{\mathrm{BLH}}$ with $\mu$ the Coulomb friction coefficient and $F_{\text {BLH }}$ the blank-holder force (Fig. $7($ a)). 


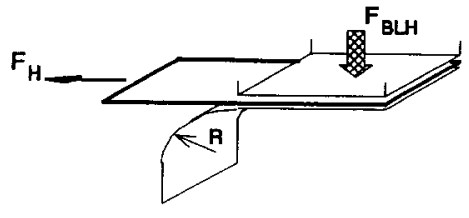

(a)

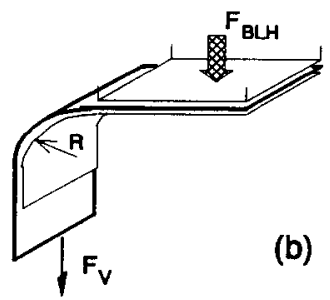

(b)

Fig. 7. Simulation of slip between the die and the blank-holder.

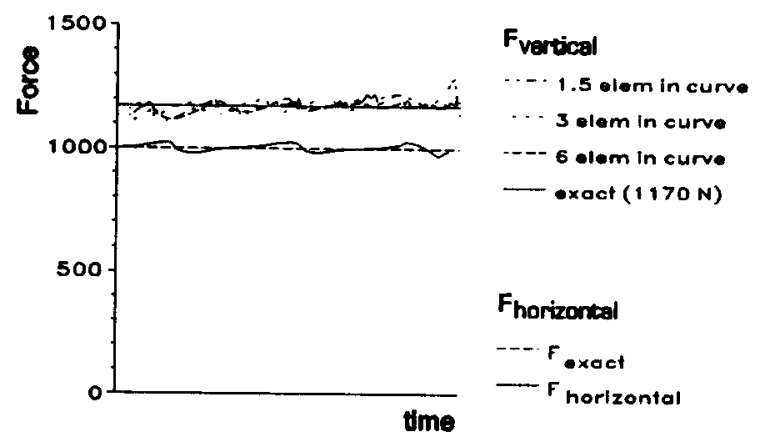

Fig. 8. Force required to pull out the strip as a function of time.

The strip can also be pulled in the vertical direction (Fig. 7(b)). If bending is neglected, the required vertical force, $F_{\mathrm{V}}$ (with $\beta$ the angle of contact) can be expressed as

$$
F_{\mathrm{V}}=F_{\mathrm{H}} \mathrm{e}^{\mu \beta}=2 \mu F_{\text {BLH }} \mathrm{e}^{\mu(\pi / 2)} .
$$

In this example, the die is modelled by a contour consisting of a vertical plane, a part of a cylinder and a horizontal plane. The blank-holder is modelled by a horizontal plane. To examine the effect which the modelling of curvatures with a limited number of elements may have, the authors varied the radius of the die, whilst keeping the dimensions of the elements unchanged.

The friction coefficient $\mu$ was taken to be $0.1, F_{\mathrm{BLH}}$ was $5 \mathrm{kN}$ and the total strip length was $50 \mathrm{~mm}$. The radius was taken such that the number of elements located in a quarter-circle was respectively $\pi / 2(\simeq 1.5), \pi(\simeq 3)$ and $2 \pi(\simeq 6)$.

The simulations were carried out in 50 steps, in the last step, the strip being nearly pulled out. The forces $F_{\mathrm{H}}$ and $F_{\mathrm{V}}$ are presented in Fig. 8. The wiggles observed in the forces in Fig. 8 are caused by geometrical non-linearities. Fig. 8 demonstrates clearly that the agreement of simulation and theoretical prediction is very satisfactory, even when a $90^{\circ}$ bend is discretized by only $1 \frac{1}{2}$ elements.

In the previous section a description was given of how to avoid a parasitic stiffness. For this purpose, a surface normal is used instead of an element normal. The effect of 
this adaptation is illustrated in Fig. 9, showing again the forces necessary to pull out the strip. Fig. 9 reveals that not only does the force $F_{\mathrm{V}}$ increase, but also that the wiggles becomes more severe, especially when a small number of elements is located in a bend.

To conclude, the parasitic stiffness has severe undesired effects which are avoided by introducing a $\mathrm{C}-1$ continuity.

\section{Geometry description}

Many CAD packages have a FEM-module with the capability to generate elements on the surface of tools. Further pre- and post-processors such as PATRAN can utilise the database of CAD packages. A method is developed to adjust the nodes of contact elements on the defined surface. This happens in such a way that the contact node moves to a location on the surface which is closest to the complementary nodal point fixed on the sheet. The adjustment takes place every increment. Surfaces can be constructed from a library of geometrical primitives such as planes, cylinders, torusses, etc. Alternatively, a surface can be defined by a collection of elements.

When following this procedure, FEM developers do not have to use Bezier curves and more exotic mathematical exercises.

\section{Examples}

\subsection{Example 1. Simulation coupelle Renault Twingo}

In co-operation with Renault $R \& D$, France, finite-element codes for deep-drawing simulations have been evaluated. Further, the effects of friction, material properties and process conditions on deep-drawing processes have been studied. Most of the interest is in automotive products, this example showing the forming of a 'coupelle' of the Renault Twingo. (A 'coupelle' is a bearing part of the suspension system in automobiles).

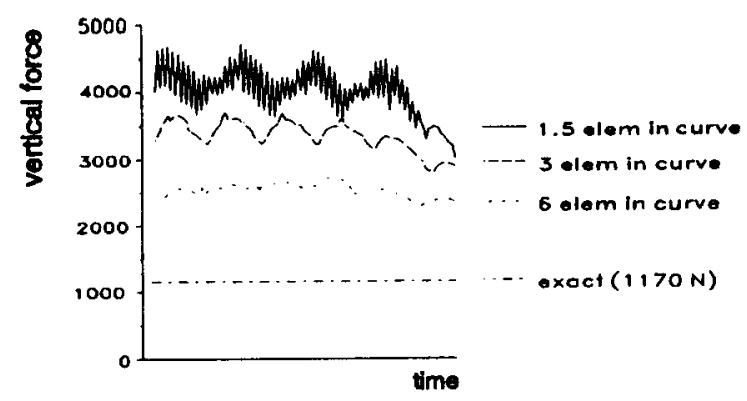

Fig. 9. Applied force with element normals rather than tool normals. 
This example shows the influence of the blank-holder force on the deep-drawing process. At first a force as applied in the press shop was applied to the blankholder and in the second case a force of $1 / 10$ of the original blank-holder force was applied.

To form the 'coupelle' the usual combination of tools is used: a punch, a matching die and a blank-holder. The tool surfaces are modelled by 3 and 4 node elements, see Fig. 10. The first calculation showed a smooth product, displayed with shaded image in Fig. 11. The simulation is performed on an IBM RS6000-320h machine and took 5 CPU hours. The second simulation predicts the occurrence of wrinkling and had severe problems in converging due to rapidly changing contact conditions. The

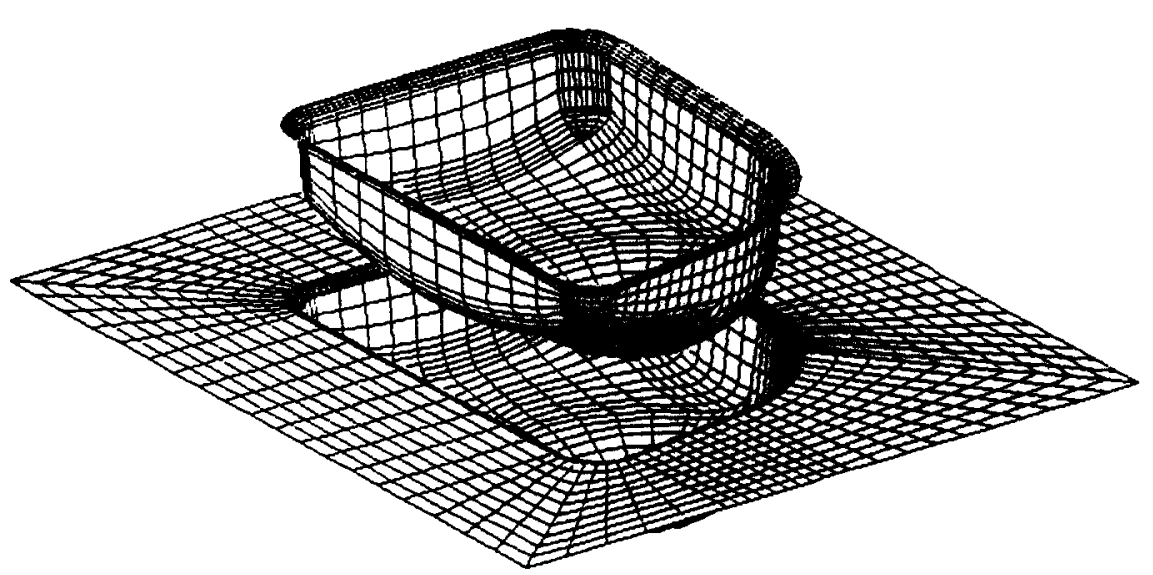

Fig. 10. Tools modelled with elements.

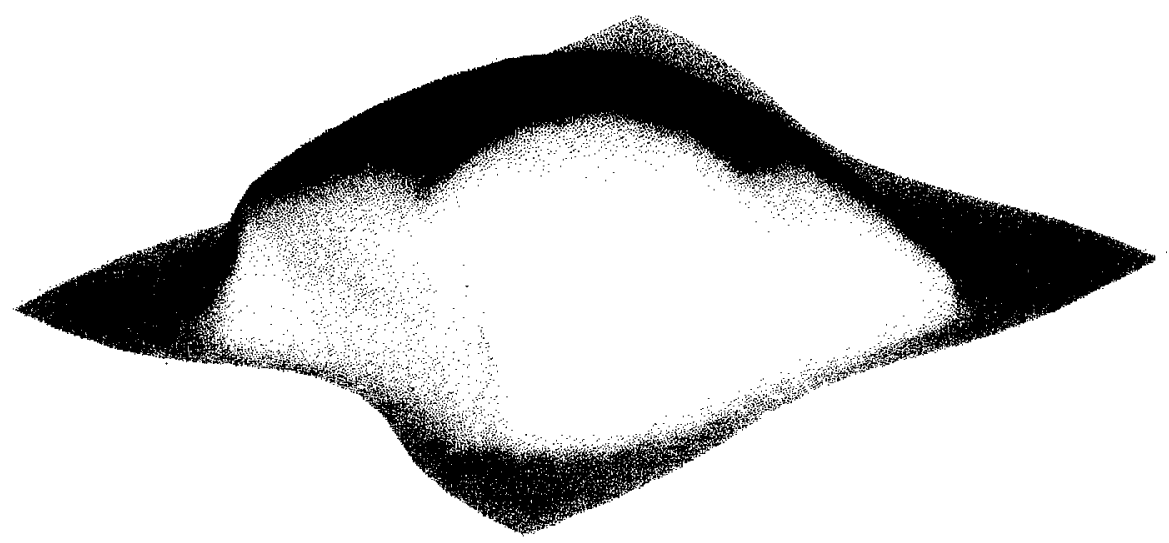

Fig. 11. Image of a product with the normal blank-holder force. 


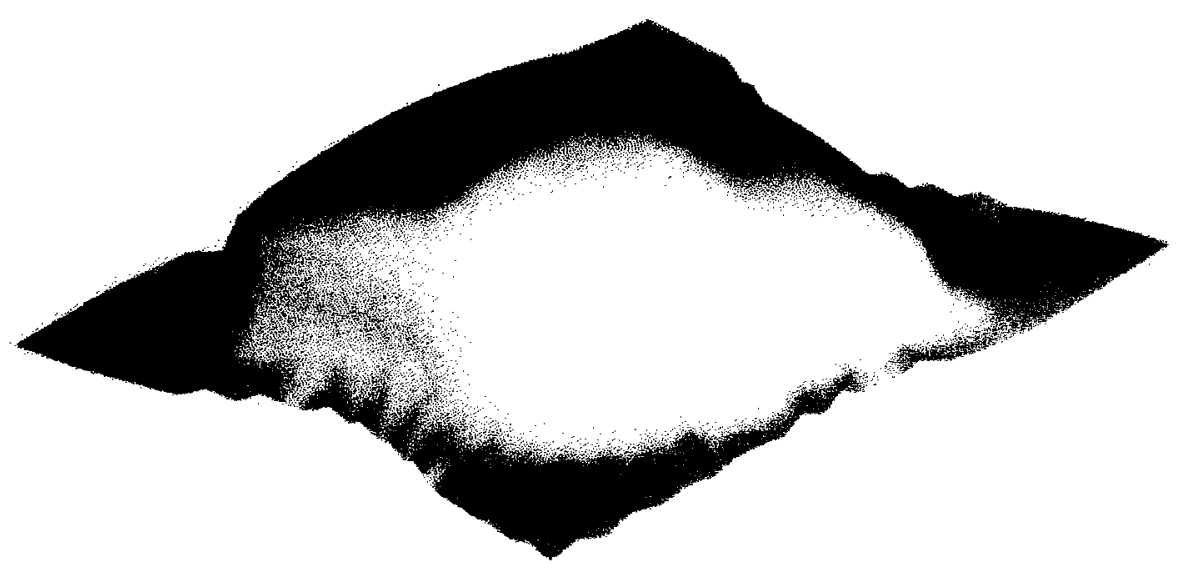

Fig. 12. Image of a product with one-tenth of the normal blank-holder force.

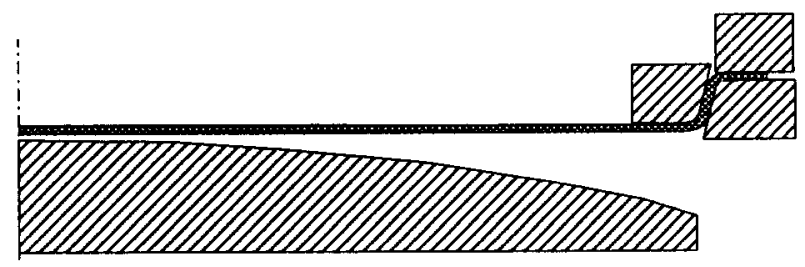

Fig. 13. Dinner-tray-like product.

calculation lasted $30 \mathrm{CPU}$ hours on the same workstation. The image is displayed in Fig. 12. The calculated thickness-distribution was compared with the measured thickness-distribution and seen to correspond closely at first glance.

\subsection{Example 2: Simulation satellite disc}

A satellite disc is formed with help of a multi-step drawing process. Since a considerable shape-accuracy is required, the spring-back of the product is of special interest. In the first step a dinner-tray-like product is formed, see Fig. 13. In the second step the blank-holder force is increased to prevent slip and the paraboloided shape is stretched into the product. Finally, the product is unloaded, whereupon some spring-back occurs.

Near the clamping zone sharp bending takes place. As shell elements behave poorly in zones which are dominated by shear, axial symmetric 4-node isoparametric bulk elements are applied. In the clamping zone 3 elements are located in the thickness direction. In the zone under the plunger, stretching dominates the deformation process and 1 element is applied in the thickness direction. In Fig. 14 the predicted spring-back is compared with the results of measurement. Finally, the punch force-displacement diagram is shown in Fig. 15. 


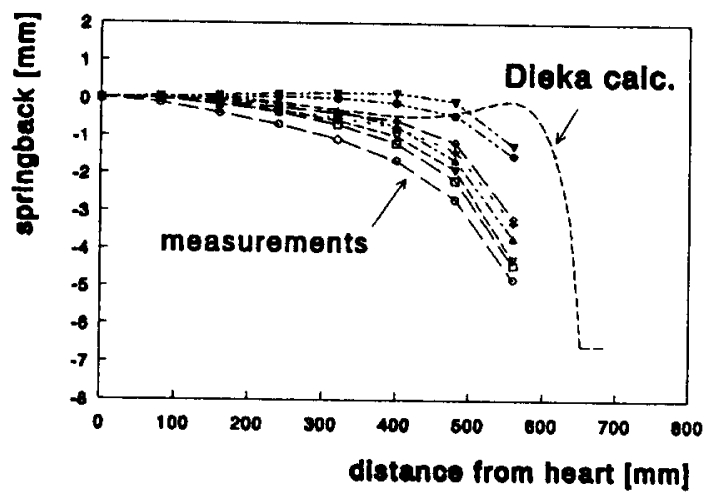

Fig. 14. Measurements and calculation.

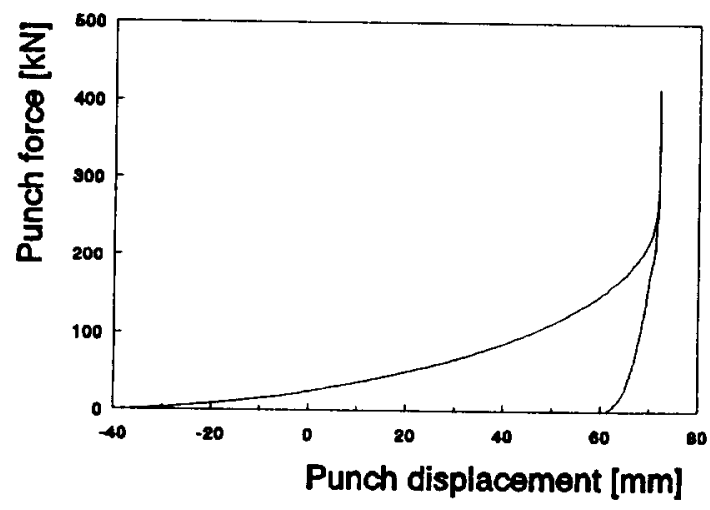

Fig. 15. Punch force-displacement diagram.

\section{Explicit solution methods}

When the problems became larger the number of degrees of freedom increased accordingly and, as a matter of fact, when employing implicit methods, the limits of computer memory are approached and acceptable computer times are exceeded. In fields of crash analysis, explicit solution methods are used commonly and this method provides opportunities to satisfy the ambition of doing simulations with a large number of elements. Elaborating on this approach an alternative procedure is proposed. The method is under development currently and only the first relatively simple test problems are reported.

The basis of the explicit method is the equation of motion. Suppose damping can be neglected, $\boldsymbol{M}$ is the lumped mass matrix, $\ddot{\boldsymbol{u}}$ is the acceleration vector and $\boldsymbol{p}$ and $\boldsymbol{f}$ are the vectors with internal and external forces respectively. In contrast with usual 
formulations a variable time step $\Delta t_{i}$ is choosen. The velocities discretized from the displacements $\boldsymbol{u}$ :

$$
\dot{u}_{n-1 / 2}=\frac{u_{n}-u_{n-1}}{\Delta t_{i}} ; \quad \dot{u}_{n+1 / 2}=\frac{u_{n+1}-u_{n}}{\Delta t_{i+1}}
$$

The acceleration can be obtained directly:

$$
\ddot{u}_{n}=\frac{\boldsymbol{u}_{n+1}-\boldsymbol{u}_{n}}{(1 / 2) \Delta t_{i+1}\left(\Delta t_{i}+\Delta t_{i+1}\right)}-\frac{\boldsymbol{u}_{n}-\boldsymbol{u}_{n-1}}{1 / 2 \Delta t_{i}\left(\Delta t_{i}+\Delta t_{i+1}\right)}
$$

When both time increments equal $\Delta t$ the well-known central difference scheme appears. The following displacement increments: $\Delta \boldsymbol{u}_{i+1}=\boldsymbol{u}_{n+1}-\boldsymbol{u}_{n}$ and $\Delta \boldsymbol{u}_{i}=$ $\boldsymbol{u}_{n}-\boldsymbol{u}_{n-1}$ and relation (17) is substituted into the equation of motion to provide the new displacement increment:

$$
\Delta u_{i+1}=\frac{\Delta t_{i+1}}{\Delta t_{i}} \Delta u_{i}+\frac{1}{2} \Delta t_{i+1}\left(\Delta t_{i}+\Delta t_{i+1}\right) M^{-1}(p-f)
$$

This procedure can be applied on contact also. The main object of a contact algorithm is to prevent penetration into a body and account for friction. The penalty method is applied frequently. At the end of a step, a check on penetration is done in the event of penetration, penetration distance $\boldsymbol{h}$ is calculated. The reaction force due to overlap can be calculated by multiplication of the overlap with the contact penalty factor. A simple test of this procedure can be carried out by dropping a mass in the initial rest state under the influence of the gravity force, Fig. 16. When no energy is lost the maximum altitude should remain unchanged. In fact, however, depending on the time step, a lost or even gain of (potential) energy is observed, as indicated in Fig. 17.

The following procedure is proposed. In the time step in which overlap starts to occur, the time and displacement increment is reduced such that the material point touches the tool. From the overlap a force in the opposite direction is applied and the material point is relocated at the tool surface. The same experiment is carried out, whereupon the advantage of this procedure is obvious, as can be seen in Fig. 18.

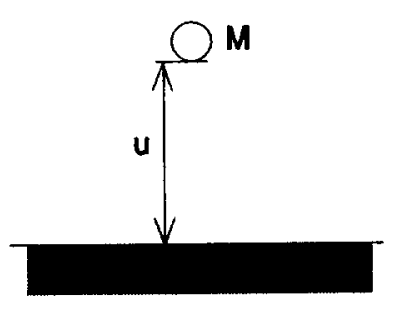




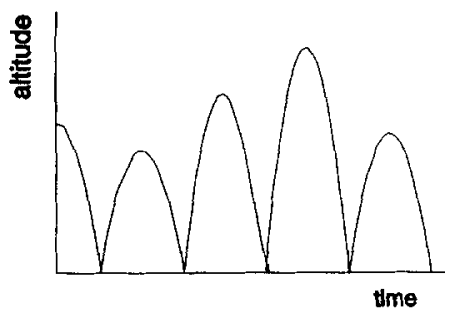

Fig. 17. Unstable modelling.

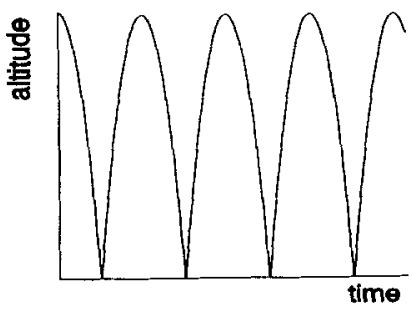

Fig. 18. Stable modelling.

\section{References}

[1] J. Huétink, Extension of anisotropic hardening elastic-plastic theory to finite strains based on visco-elastic finite strain theory, IUTAM Symp., Hannover, Germany, 1991.

[2] P.T. Vreede, A finite element method for simulations of 3 dimensional sheet metal forming, Dissertation University of Twente, The Netherlands, 1992.

[3] R.D. Krieg, A practical two surface plasticity Theory, J. Appl, Mech., 3 (1975) 641-646.

[4] F. Ellyin and Z. Xia, A rate independent constitutive model for transient non-proportional loading, J. Mech. Phys. Solids, 37(1) (1989) 71-91.

[5] A. Phillips and C.W. Lee, Yield surfaces and loading surfaces, Experiments and recommendations, Int. J. Sol. Struct., 15 (1979) 715-729.

[6] H. Vegter, On the plastic behaviour of steel during sheet forming, Dissertation University of Twente, 1991.

[7] P.T. Vreede, M.F.M. Louwes, J. Huétink and N.A.J. Langerak, Contact behaviour modelled with interface elements and tool description, in: J.L. Chenot, R.D. Wood, O.C. Zienkiewicz (Eds.), Numiform '92, (Eds.), A.A. Balkema, Rotterdam. The Netherlands, 1992.

[8] W.C. Emmens, The influence of surface roughness on friction, Proc. 15th Biennal Congress, IDDRG, Dearborn, USA, 1988.

[9] W.C. Emmens and G. Montfort, The influence of process conditions and surface characteristics on friction at low pressure, Adv. Technol. Plast., 3 (1990) 1277-1284.

[10] E. Nakamachi, Deep drawing analysis of square cups with coated, rate-sensitive, steel sheets, in: D.R. Owen, E. Onate, E. Hinton (Eds.), Complas, Pineridge Press, Swansea, UK, 1991. 